\title{
Effect of Using the Paperless Partograph Versus the original Partograph on Labor Outcomes in Women's Health Hospital.
}

\author{
Abeer A. Mohammed ${ }^{1}$, Howieda A. Fouly ${ }^{2}$, Ahmed M . Abbas ${ }^{3}$, Manal F. Mostafa ${ }^{4}$ \\ 1. Nursing Specialist at Women's Health Hospital in Assiut University, Egypt. \\ 2. Lecturer of Obstetric \& Gynecologic Nursing, Faculty of Nursing- Assiut University, Egypt. \\ 3. Lecturer of Obstetrics \&Gynecology, Faculty of Medicine -Assiut University, Egypt. \\ 4. Professor of Obstetrics \& Gynecological Nursing, Faculty of Nursing- Assiut University, Egypt .
}

\begin{abstract}
Background: The paperless partogram refers to monitoring progress of labor and arriving at an accurate decision for intervention to ensure safe delivery. It needs no graph paper, no extra time to do it. Aim: To evaluate the efficacy of using the paperless partograph versus the original partograph on labor outcomes in Women's Health Hospital. Sampling : Random sample of 370 participants. Setting: The study was conducted from April 15 th , 2016 to September 30 th , 2016 in Women Health Hospital, Assiut University, Egypt, at reception (emergency ) unit. Design method: quesi-experimental design. Study subjects: This study was randomly assign women into 2 groups. Group (1): was allocated to modified WHO partograph and Group (2): was allocated to paperless partograph. Results : Comparison between studied groups as regards time taken for first stage and second stage revealed a statistical significant difference at $\mathrm{P}$. value $(<0.017 \&<0.001)$ respectively. For mode of delivery revealed a statistical significant difference at $\mathrm{P}$. value $(<0.0152)$. For postpartum complications revealed a statistical significant difference at $\mathrm{P}$. value $(<0.003)$. Conclusion: The Paperless partograph was effective positively than the WHO modified partograph in the management of labor. Recommendations: This method should be implemented as essential part of care in all health facilities, and used by all clinical training sites to give trainees (doctors and nurses midwives) an opportunity to use it.
\end{abstract}

\section{Keywords: Partograph, Labor, WHO Modified Partograph \& Paperless Partogram.}

\section{Introduction}

Partograph is a paper chart on which remarks in labor are recorded in a graphic design to provide an outline of labor, pointing to alert midwives and obstetricians to deviance in labor progress as well as maternal or fetal wellbeing. (Lavender \& Hart in 2009).

However, there are factors have been implicated to squat use of partograph such as deficiency of attentiveness and appropriate training, decrease handiness of partograph, negative awareness of the partograph, great patient load, insufficient staff at the health institutions, shortage of direction, and negative attitudes among some of the health workers Continuous monitoring of labor and provision of rapid care to deal with problems are most crucial for preventing adverse obstetric outcomes related to childbirth. Debdas argues that the WHO partograph fails to meet the present requirements and proposes the paperless partograph (Debdas,2008).

Therefore to improve using of partograph, there is a simple paperless partograph could be used. In which is, less graph, no time consuming, only comprises the calculation of an expected time of delivery (ETD) (Mishra, 2014). Thus, paperless partograph is a method used for watching the progress of labor before delivery, and reaching at the accurate time for safe delivery. Dissimilar to the current practice of writing the expected date of delivery (EDD) and other considerations that extend labor and delivery time, the method only records the expected time of delivery (ETD), the alert ETD and action ETD for accurate and precise intervention (Mitchell,2010).

Role of Nurse in using of Partograph

Nurse plays a vital role in providing care during labor. She is the one who works to immediate environment with the patient and hence there she has all the opportunity to identify the needs and problems of the patient to improve the quality of life. A partograph is a graphical presentation of a woman's progress of labor. Once the woman has true signs of labor, the midwife initiates the use of the partograph to record her findings. The partograph was endorsed and modified by the World Health Organization (WHO) between 1990 and 2000 to monitor the fetal and maternal wellbeing during the active stage of labor. Monitoring help the midwives and the mother in achieving spontaneous vaginal delivery with low risk of both morbidity and mortality. Furthermore, accurate partograph record keeping enables an effective communication between healthcare professionals who manage women in labor. Documentation and record keeping have always been integral to midwifery practice and continue to be so (Debdas, 2008). 
Midwives or obstetric \& gynecologic nurses working in the labor ward work directly with the partograph. Once the women transferred to labor ward and reached to the active phase of labor. They expected to be monitored on the partograph by Obgyn nurses to show progress of labor as well as fetal and maternal condition. Also, the obgyn nurses expected to analyses the partograph in retrospect to establish if there were any significant consequences during labor that would influence the management of the mother and baby post-delivery. Partograph significantly improved the outcome of labor in both maternal and neonatal perspectives and therefore recommended that it should be used in all maternity units. The charts that were retrospectively analyzed showed that significantly more recordings had correct admission times and the name of the patient since the maternity case record has the client's name on the cover (Fraser et al., 2009).

\section{Significance of the study}

Preventing prolonged labor is a key strategy for reducing maternal and neonatal death. Once cervical dilation reaches 4 centimeters and contractions happen every ten minutes, a woman is considered to be in the active stage of labor. If this stage lasts too long, the woman faces higher risk of postpartum hemorrhage, sepsis, uterine rupture, and death. Likewise, prolonged labor places neonates at higher risk for anoxia, infection, and intra-partum death (Debdas, 2008).

The paperless partograph refers to monitoring progress of labor within a precise decision for intervention to ensure safe delivery. Sequentially, reducing maternal and neonatal death by preventing prolonged labor. It needs no graph paper, no extra time to do it and uses the routine that the nurses are already used. Paperless partograph guide the provider about the place to go, number of hours, time to terminate labor and exact time to transfer a woman to some higher center with Cesarean capability (Debdas, 2008)

\section{Aim of the study}

To evaluate the efficacy of using the paperless partograph versus the original partograph on labor outcomes in Women's Health Hospital

The study hypothesis

$\mathbf{H}^{\mathbf{1}}$ :The use of the paperless $\mathrm{p}$ artograph will be easier and effective design for labor management and outcomes.

$\mathbf{H}^{\mathbf{0}}$ :The use of paperless partograph will not be easier and effective design for labor management and outcomes.

\section{Materials \& Methods Study setting}

The study was conducted from April 15th, 2016 to September 30, 2016 in Women Health Hospital, Assiut University, Egypt, at reception (emergency) unit, The reception unit consist of (5) rooms one them is examination room with a capacity of (3) bed, one other is prenatal room with a capacity of (4) bed, other is postpartum room with a capacity of (8) bed and two other are operational room. Work in it (10) diploma nurse and (1) head nurse.

\section{Sampling method}

(Randomized controlled trial).

Study design

Quasi-experimental design.

Study subjects

This study was randomly assign women into 2 groups.

Group (1): was allocated to modified WHO partograph

Group (2): was allocated to paperless partograph.

\section{Sample size}

The sample size was calculated using the formula $\mathrm{N}=(\mathrm{Z} \alpha+\mathrm{Z} \beta) 2 \times 2 \mathrm{p}(1-\mathrm{p}) \div \mathrm{d} 2$, taking the level of significance as $5 \%, Z \alpha=1.96$ and power of the test as $80 \%, Z \beta=0.84$. $p=0.113$. A sample size of 165 in each group was calculated. Taking in account a $10 \%$ rate of drop-out cases, a total of 370 women will be studied (185 in each arm).

\section{Patients}

The women was enrolled in the study $(\mathrm{N}=370)$. Inclusion criteria

Age: 18 to 40 years, gestational age 38-42 weeks , singleton pregnancy, vertex presentation, and women who will accept to participate in the study.

Exclusion criteria: Malpresentation, induced labor, Multiple pregnancy and Medical diseases with pregnancy

\section{Primary Outcome}

Number of successful vaginal deliveries

Secondary Outcomes:

- Intrapartum maternal of fetal distress

- Duration of labor

- Rate of cesarean section.

Asessment

Assess the outcome of maternal and newborn condition as mode of delivery, birth outcome, birth weight, Apgar score, and any complication may raise for mother or newborn.

\section{Tools of the study:}

Tool I: A Structured interviewing questionnaire: would be developed by the investigator and used to collect the socio-demographic data, obstetric history. Tool II: Modified WHO Partograph Model.

Tool III: Paperless partograph Model. 
The paperless partograph is a simple, inexpensive tool to provide a continuous pictorial overview of labor. It is a technique used for labor management, only after the cervical dilatation reach $4 \mathrm{~cm}$ or more for monitoring the progress of labor until delivery, and arriving at the accurate time to intervene for ensuring a safe delivery. It used by clinicians or nurses through calculation of two times, an ALERT ETD (estimated time of delivery) and an ACTION ETD. The ALERT calculation uses Friedman's widely accepted rule that the cervix dilates $1 \mathrm{~cm}$ per hour while a woman is in active labor (Friedman's 1955). Once cervical dilation reaches 4 centimeters and contractions happen every ten minutes, a woman is active stage of labor. The clinician simply adds 6 hours to the time at which the woman becomes dilated to $4 \mathrm{~cm}$ to find the ALERT ETD. (when cervical dilation is at $10 \mathrm{~cm}$ ). the clinician adds 4 hours to the ALERT ETD to get the ACTION ETD. both ETDS should be written in big letters on a woman's case management questionnaire, the ACTION ETD circled in red. (Debdas,2008).

\section{Operational design}

This design involves :Pilot study

pilot study was done on 40 women $(10 \%$ of total sample).

Field work \& Intervention.

The field of work: is divided into two phases: Phase 1: Women intervention during labor.

- Greeted and introduced herself to each woman and explained the aim of the study and obtaining the oral informed consent before the enrollment in the study. study sample through questionnaire sheet provided by the researchers to collect the basic data (Name, Age, Residence, Education, Gravidity, Parity, live male, live female, still birth, gestational age and cervical dilatation at admission).

- The investigator applied the randomized selection of the participant as the following: gave the participant an envelope contains two choice of (A or B) labeled papers then according to her choice she was engaged in the group: Group (A) means WHO modified partograph and group B used paperless partograph. The investigator used the WHO modified partograph to follow-up group A. Then the other group (B) were follow up by using paperless technique which developed by Debdas 2008. It needs no graph paper, no extra time to do it and uses the routine that the nurses are already used

- It gives the two basic data on which the partogram works namely (The Alert line \&The Action line). This method involves only finding Expected Time of Delivery (ETD) and then adhering to that without any recurrent plotting of data and its interpretation. and the paperless partogram model was used in labor management and outcome of labor was detected.

- Each woman was individually interviewed using the previously mentioned study tools from active phase until delivery and all data recorded in the paperless partogram model. Calculate two times,an ALERT ETD(estimated time of delivery)and an ACTION ETD calculation uses friedman's widely accepted rule that cervix dilates $1 \mathrm{~cm}$ per hour while a women is in active labor.Simply adds 6 hours to the time at which the women becomes dilated to $4 \mathrm{~cm}$ to find the ALERT ETD (when the cervical dilatation is at $10 \mathrm{~cm}$ ).Adds 4 hours to the ALERT ETD to get the ACTION ETD. Both ETDS should be written in big letters on a women's case management sheet, the ACTION ETD circled in red.

Phase 2: Assessment of maternal and neonatal outcomes:

\section{Maternal Outcomes \\ Primary Outcome}

Number of successful vaginal deliveries Secondary Outcomes

Intrapartum maternal of fetal distress

Duration of labor

Rate of cesarean section

neonatal outcomes: After the baby was born assessed Apgar score for baby at 1 minute and at 10 minute after that weighted the baby and assessed any complications with the baby.

\section{Administrative design}

Before conducting the study an authorized permission was obtained by submission of an official letter from the Faculty of Nursing -Assiut University to the head of department of obstetrics and gynecology in (Women Health Hospital -Assiut University) to obtain the authorization for data collection.

\section{Ethical consideration}

Research proposal was approved from Ethical Committee in the Faculty of nursing. The study was follow common Ethical principles in clinical research. The aim of the study was explained to every woman before participation, and voluntary participation was emphasized and an oral consent was obtained, however; they had the right to withdraw from the study at any time. Data collection was anonymous, and confident iality of the data was secured.

There was no risks for study subject during application of the research. The. Study subject privacy would be considered during collection of data. A written consent will be obtained from women or guidance that are willing to participate in the study. 


\section{Statistical design}

The data were tested for normality using the Anderson-Darling test and for homogeneity variances prior to further statistical analysis. Categorical variables were described by number and percent $(\mathrm{N}$, $\%)$, where continuous variables described by mean and standard deviation (Mean, SD). Chi-square and fisher exact tests used to compare between categorical variables where compare between continuous variables by unpaired t-test. A two-tailed p 0.05 was considered statistically significant. All analyses were performed with the IBM SPSS 20.0 software.

\section{Result}

Table (1): Comparison between studied groups as regards obstetric history.

\begin{tabular}{|c|c|c|c|c|c|}
\hline \multirow{3}{*}{ Obstetric History } & \multicolumn{4}{|c|}{ Study participants $\mathbf{N}=\mathbf{3 7 0}$} & \multirow{3}{*}{ P. value } \\
\hline & \multicolumn{2}{|c|}{ Paperless partogram } & \multicolumn{2}{|c|}{$\begin{array}{c}\text { WHO Modified } \\
\text { partograph }\end{array}$} & \\
\hline & No. & $\%$ & No. & $\%$ & \\
\hline \multicolumn{6}{|l|}{ Age } \\
\hline Range & \multicolumn{2}{|c|}{$19-40$} & \multicolumn{2}{|c|}{$18-40$} & \multirow{2}{*}{0.295} \\
\hline Mean \pm SD & & & & & \\
\hline \multicolumn{6}{|l|}{ Parity } \\
\hline Range & \multicolumn{2}{|c|}{$0-9$} & \multicolumn{2}{|c|}{$1-10$} & \multirow{2}{*}{0.421} \\
\hline Mean \pm SD & \multicolumn{2}{|c|}{$2.6 \pm 1.8$} & \multicolumn{2}{|c|}{$2.6 \pm 1.7$} & \\
\hline \multicolumn{6}{|l|}{ Live male } \\
\hline Range & \multicolumn{2}{|c|}{$0-4$} & \multicolumn{2}{|c|}{$1-4$} & \multirow{2}{*}{0.861} \\
\hline Mean \pm SD & \multicolumn{2}{|c|}{$1.6 \pm 0.8$} & \multicolumn{2}{|c|}{$1.6 \pm 0.8$} & \\
\hline \multicolumn{6}{|l|}{ Live female } \\
\hline Range & \multirow{2}{*}{\multicolumn{2}{|c|}{$\frac{0-4}{18+1}$}} & \multirow{2}{*}{\multicolumn{2}{|c|}{$\frac{1-6}{17+1}$}} & \multirow{2}{*}{0.972} \\
\hline Mean \pm SD & & $1.8 \pm 1$ & & $1.7 \pm 1$ & \\
\hline
\end{tabular}

Table (2): Comparison between studied groups as regards current labor/delivery.

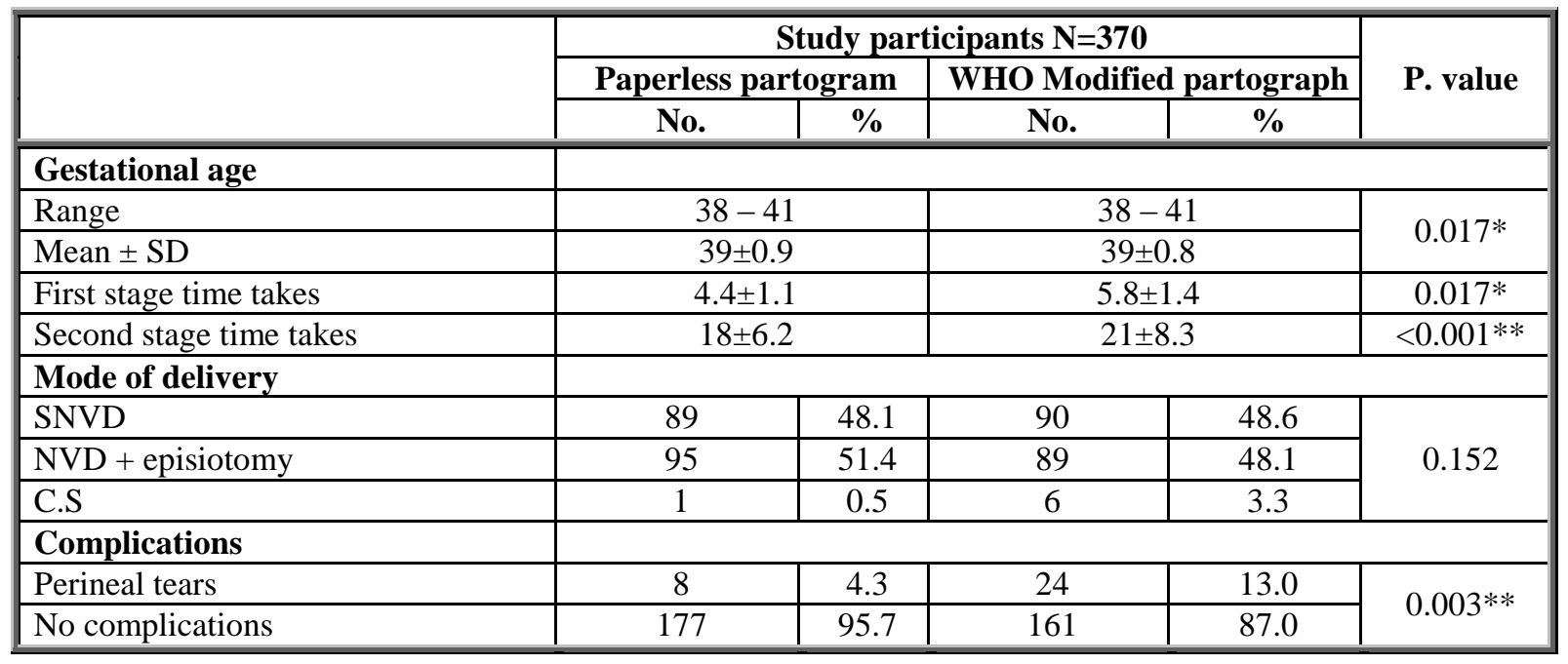

Table (1): No statistically significant difference between studied groups as regard obstetric history . Table (2): shows Comparison between studied groups as regards current labor/delivery data revealed a statistical significant difference for gestational age at $\mathrm{P}$. value( 0.017). Regarding time taken for first stage and second stage revealed a statistical significant difference at P. value ( 0.017 \& $0.001)$ respectively. For mode of delivery revealed a statistical significant difference at $P$. value( 0.0152$)$. For postpartum complications revealed a statistical significant difference at $P$. value ( 0.003). 


\section{Discussion}

Monitoring labor can be undertaken in various ways. One method commonly used in developing countries is the partograph (or partogram). The partogram is a tool that enables midwives and obstetricians to record maternal and fetal observations. WHO has recommended universal use of a partogram during labor to aid in clinical decision-making (Banerjee $\boldsymbol{\&}$ Sharma, 2015).

The WHO recommendation has not changed despite a 2009 Cochrane review of five randomized controlled trials (including both high- and lowresource countries .The paperless partograph is a low-skill method for preventing abnormal labor (Agarwal et al., 2013), It is designed to monitor not only the progress of labor, but also the condition of the mother and the fetus during labor. Paperless partogram needs no graph paper, no extra time to do it and uses the routine that the nurses are already used to. It gives the two basic data on which the partogram works namely (The Alert line and The Action line). This method involves only calculate alert and action times by adding six hours to the time when a woman reaches $4 \mathrm{~cm}$ of dilatation (alert line) and adding four hours to the alert time (action line); based on rational that cervix should dilate $1 \mathrm{~cm}$ per hour between $4 \mathrm{~cm}$ and $10 \mathrm{~cm}$. If there is no birth at alert time, refer to care and if no birth by action line, immediate delivery (Technologies for Health Consultative Meeting, 2015). Very little researches done to test the effectiveness of paperless and its acceptance from heath care providers (Nurses midwives and Obstetricians); so it is important to conduct the present study that aimed to evaluate the effect of use the paperless partogram on outcome of labor.

The base line data of women revealed that the present study enrolled 370 laboring women, their ages ranged from 19 to 40 years old with average Mean \pm SD 25.1 \pm 5.4 years. These results is slightly similar to the finding conducted in Labor ward at Maternity Hospital affiliated to Ain Shams University, Egypt. .which used the paperless partogram for out of 100 laboring women who participated in the study which revealed that their ages ranged from 18 to 35 years with average 25.6 \pm 5 years. The parity of participants ranged from 0-9 with mean \pm SD $2.6 \pm 1.8$. These results is slightly similar to Fatouh \& Ramadan, (2015) in Helwan University Egypt. which the parity was $2 \pm 1.3$.

The gestational age at onset of labor ranged from 37 to 40 weeks with mean $\pm \mathrm{SD}=39.1 \pm 1.86$. these results is slightly similar to (Fatouh \& Ramadan (2015) which gestational age of participants ranged from $38-41$ weeks with Mean \pm SD 39 \pm 0.9 .
As regard to the First stage time takes ranged with Mean \pm SD 4.4 \pm 1.1 hours and Second stage time takes with Mean SD $21 \pm 8.3$ minutes. These results were nearly corresponding to the study findings conducted by Agarwal et al., (2013) which used the paperless partogram for the management of labor, out of 91 participants, the study revealed that the mean duration for delivery after Alert ETD was 4.3 hours, The mean duration for delivery after Alert ETD was $4.7 \pm 1.9$ hours in Primigravida and $3.7 \pm$ 1.8 hours in multipara, however, these differences were not statistically significant ( Agarwal et al., 2013), and was similar to the WHO recommendation for partogram with a four-hour action line denoting the timing of intervention for prolonged labor (Banerjee \& Sharma, 2015).

As regard to the First stage time takes ranged with Mean \pm SD 4.4 \pm 1.1 hours and Second stage time takes with Mean SD 21 \pm 8.3 minutes these findings were nearly corresponding with other study which used the paperless partogram for the management of labor (Fatou, Ramadan 2015) the study revealed that the total mean duration for delivery after Alert ETD was $3.4 \pm 2.1$ hours, the average time for delivery among the Primigravida women after the Alert ETD was $3.5 \pm 2.1$ hours (ranged from 1 to 7 hours) and multiparous women after the Alert ETD was $3.3 \pm 2.1$ hours (ranged from 25 minutes to 6 hours).

The present study revealed that the most of the women $48.1 \%$ were spontaneous normal vaginal delivery, $51.4 \%$ were normal vaginal delivery with episiotomy and $0.5 \%$ were caesarian section. These findings were nearly corresponding with Fatouh \& Ramadan, 2(015) which used the paperless partogram for the management of labor revealed that the most of the women had normal vaginal delivery $(88 \%)$, whereas only $12 \%$ of them had caesarean section. These findings were nearly corresponding with other study which used the paperless partogram for the management of labor; labor was induced only in 13\% of the cases (Agarwal \& Sharma, 2013). which shown to be effective in preventing prolonged labor, in reducing Cesarean Section. The most of cases in the current study not reach to action line and those who reach to it were given the appropriate care in suitable time, so none of them complicated by prolonged labor.

As regard to the neonatal outcome; the average Apgar score after 1 minute and 5 minutes were $(8.7 \pm 0.4 \& 99.9 \pm 0.1$ respectively). This result was interpreted by there was no newborn need to admit to Neonate Intensive Care Unit (NICU) or need ventilation. This result revealed the positive effect of paperless partogram on neonatal outcome but this result need to be proved by other researches; as 
minor studies focused on the effect of paperless partogram on labor outcome, unfortunately; none of studies effect on neonatal outcome (Debdas, 2008, Elizabeth, 2010, Mitchell, 2010, Agarwal et al., 2013).

As regard to the neonatal outcome; all the newborns weights were within normal. The average weight of the new-born ranged from $2-4 \mathrm{~kg}$ with Mean \pm SD3.2 \pm 0.6 which the live birth $0-4$ live male with mean \pm SD1.6 \pm 0.8 and $0-4$ were live female with mean \pm SD $1.8 \pm 1$ of participants. and the average Apgar score after 1 minute and 5 minutes were $(8.7 \pm 0.4 \& 99.9 \pm 0.1$ respectively). These results were nearly corresponding to the study findings conducted by Fatouh \& Ramadan, (2015) the average weight of the new-born ranged from 3.6 $\pm 0.4 \mathrm{~kg}$ and $51 \%$ of them were females and $49 \%$ males. The average Apgar score after 1 minute and 5 minutes are $(7.3 \pm 0.9 \& 9.4 \pm 0.9$ respectively).

The use of paperless partogram in labor management very simple and not needs graph paper or extra time to do it, but the WHO partogram is very complicated and require more training before using it. This finding was supported by Debdas, (2008) which argues that the partogram of WHO fails to meet the organization's own requirements for appropriate technology and has not been adapted to local needs, was not acceptable to those who use it and too complicated for many skilled birth attendants - many of whom have not received higher education.

Also it was reported that despite the proven effectiveness of traditional partogram, members pointed out they were not frequently used at all health care facility level (Debdas, 2008). Additionally; some argue that use of the partogram is complex and too time consuming for effective use in low-resource countries that have been inadequate health care staffing (Technologies for Health Consultative Meeting, 2015).

Understanding barriers facing partograph use, and the limitations facing low-resource areas, is an essential first step in effectively addressing the low rates of partograph completion and use. Effective partograph initiatives need to be cost-effective, intuitive, need to promote training and ongoing education, and must work within the complex set of issues contributing to staff and supply shortages in developing countries. The Paperless partogram attempts to provide a low-cost, intuitive solution to many of the problems that facing effective partograph use in the developing world. So the findings of present study was supported the research hypothesis which the use of the paperless partogram would be easy and effected design for the management and outcome of labor .

\section{Conclusion}

Based on the study findings; it is concluded that

- The paperless partograph is a convenient in the management of labor and shows to be an effective in preventing prolonged labor, and in improving the neonatal outcomes.

- When clinicians changed shifts ensuring that the women continues for monitoring in prolonged labor.

- The paperless partogram illustrates the potential for about 20 seconds and two time stamps, to help save the lives of mothers and babies.

- They will help in reducing maternal mortality, without any additional cost.

\section{Recommendations}

Based on the result of the present study, the researcher suggested the following recommendations

1. This method should be implemented as essential part of care in all health facilities, used by all clinical training sites to give trainees (doctors and nurses midwives) an opportunity to use it.

2. Ministry of Health should encourage active participation in activities to increase use of this tool.

3. Further studies should be done in large different health facilities (particularly in areas with high workload of patients and shortage of manpower) for focusing on the perceptions of nurses about the use of the paperless partogram.

\section{References}

1. Agarwal, K., Agarwal, L., Agarwal, V., Agarwal, A., Sharma, M., (2013): Evaluation of Paperless Partogram as aBedside Tool in the Management of Labor. Journalof Family Medicine and Primary Care.;2(1):47-9.

2. Dangal, G., (2014): Preventing Prolonged Labor by Using Partograph. The Internet Journal of Gynecology and Obstetrics..7 (1). 1: 4

3. Debdas, A., (2008): Paperless Partogram. 41st Annual Scientific Session 2008; Sri Lanka college of Obstetrics and Gynecologists. SLJOG.;30(1):124.

4. Fatouh E., Ramadan S., (2015): Effect of using Paperless Partogram on the Management and Outcome of Labor and the Nurses' Opinion. Journal of Education and Practice.;6(8):17-23. 
5. Intrapartum care: care of healthy women and their babies during childbirth (2014): NICE guidelines [CG190].. Retrieved 6 December 2014.

6. Lavender, T., Hart, A., Smyth, R., (2009): Effect of Partograph use on outcome for women in spontaneous labor at term. Cochrane Database Systemic Reviews, Issue 1. The Cochran collaboration. Published by John Wiley and sons ltd.

7. Lavender, T., Hart, A., Smyth, R., (2013): Effect of partogram use on outcomes for women in spontaneous labor at term.

8. Lavender, T., Hart, A., \& Smyth, R., (2008): Effect of partogram use on outcomes for women in spontaneous

9. Mitchell, K., (2010): The Paperless Partogram: The 20-Second Tool for Preventing Prolonged Labor.

10. Orhue, A., Aziken, M., Osemwenkha, A., (2012): Partograph as a tool for team work management of spontaneous labor. Niger J Clin Pract.;15:1-8.

11. Penumadu, K., Hariharan, C., (2014): Role of partogram in the management of spontaneous labor in primigravida and multigravida. Int J Reprod Contracept Obstet Gynecol.

12. World Health Organization, (2014): Effect of partogram use on outcomes for women in spontaneous labor atterm page.

13. World Health Organization, (1994): partograph in management of labor. Lancet ;343:1399-404.

14. World Health Organization, (1988): The partograph. A Managerial tool for the prevention of prolonged labor. In: WHO, eds. Section 1: The Principle and Strategy. WHO Document Number: WHO/MCH/88.3. Geneva. 